\title{
Lower pole renal cut injury due to the iliac wing fracture: $A$ rare case report
}

\author{
Çaglar Yildirim, Özgür Haki Yüksel, Serkan Akan, Ahmet Ürkmez, Ayhan Verit \\ Fatih Sultan Mehmet Research \& Training Hospital, Dept. of Urology, Istanbul, Turkey
}

\begin{abstract}
Summary The most frequent causes of blunt genitourinary injuries are falls from heights, motor vehicle accidents and sports injuries. Firearm injuries and penetrating stab wounds are also frequently encountered. Skeletal system traumas in the vicinity of the urogenital system can cause urological organ injuries. Though rarely, renal traumas can be dependent on the kinetic energy of the trauma and the retroperitoneal movement capacity of the kidneys and cannot be explained with the proximity of the kidney to the skeletal system. In cases with high-energy decelerations, renal pedicle and ureteropelvic junction traumas are more frequently observed. Herein, we presented a grade 3 left kidney lower pole injury developed secondary to A2 type pelvic fracture following a high energy deceleration trauma. It should not be forgotten that especially in this type of fractures, injuries of the lower renal pole can occur.
\end{abstract}

KEY WORDS: Lower pole renal trauma; Iliac wing fracture.

Submitted 12 January 2015; Accepted 15 March 2015

\section{INTRODUCTION}

Urinary system injuries occur in $10 \%$ of the trauma patients. Kidney injuries are seen in $1-5 \%$ of all trauma patients and $80-90 \%$ of them are blunt traumas. In trauma patients, isolated urogenital injuries are very rare (1). They are observed as components of multiple traumas especially as a result of falls from a height and traffic accidents (2).

Urogenital injury in blunt traumas can occur as a primary impact of the trauma and secondary to injuries of the adjacent organs exposed to trauma. In this context, incidence rates of urogenital trauma in patients with pelvic fractures have been estimated as 3.5 and $5 \%$ in the female and male patients, respectively. Among them most frequently bladder, urethra and corpus cavernosum injuries have been observed.

Bladder rupture demonstrates similar distribution (median, 3\%) among men and women. Urethral injuries are seen at a rate of $1.54 \%$ in men, while its incidence is lower in women $(\approx 0.15 \%)$ (3).

Iliac wing fractures are not frequently cited in the literature. These fractures generally occur as a result of exposure to direct blow and they maintain their stability at a vertical and rotational plane. Since isolated pelvic ring fractures do not disrupt pelvic ring stability, they can be interpreted as benign injuries. However, potentially lifethreatening critical abdominal injuries with serious vascular or neurological wounds should be also contemplated (4).

We presented a patient with multiple traumas secondary to a fall from a height which included an advanced case comminuted fracture of the iliac wing with secondary lower pole injury of the kidney. Based on our literature review, ours is the first case report on renal injury secondary to an iliac wing fracture.

\section{Case report}

A 33-year-old obese (BMI: 32) female patient was brought into the emergency service after a fall from a height. Her first physical examination revealed a moderate-bad health state with a Glasgow coma score of 10 (E2M5V3). On inspection diffuse dermabrasion, edema and ecchymosis on left side of her pelvis and an open fracture line on her left ankle were noted. Bloody urine was draining from her urethral catheter. Abdominal guarding and rebound were not detected during physical examination. On her left side costovertebral angle tenderness was elicited. Arterial blood pressure (ABP) was: $80 / 50 \mathrm{mmHg}$ and pulse rate was 110/min bpm. At her admission hemoglobin and hematocrit values were 11 gr/dl and 34\%, respectively but they declined rapidly. Indeed one hour after her referral $\mathrm{Hb}$ and $\mathrm{Hct}$ values were $6.6 \mathrm{gr} / \mathrm{dl}$ and 20\%, respectively. Arterial blood gas pH 7.2 and serum creatinine $1.06 \mathrm{mg} / \mathrm{dl}$ values were also measured.

Computed tomography obtained in the emergency service revealed the presence of multi fragmented displaced fractures of the right and left inferior and superior pubic rami, right acetabulum, right iliac wing and left femur neck. In addition, Grade 3 laceration of the lower pole of the left kidney with subcapsular hematoma in the lower pole of the left kidney with its largest diameter of $8 \mathrm{~mm}$ were noted (Figure 1 and 2).

Orthopedicians scheduled the patient for surgery, however rapid deterioration of patient's health state necessitated her treatment to be made in the intensive care unit 2 hours after her admission. Despite massive transfusion of blood and an inotropic solution, her hypotensive state did not improve and she was lost because of hypovolemic shock. 

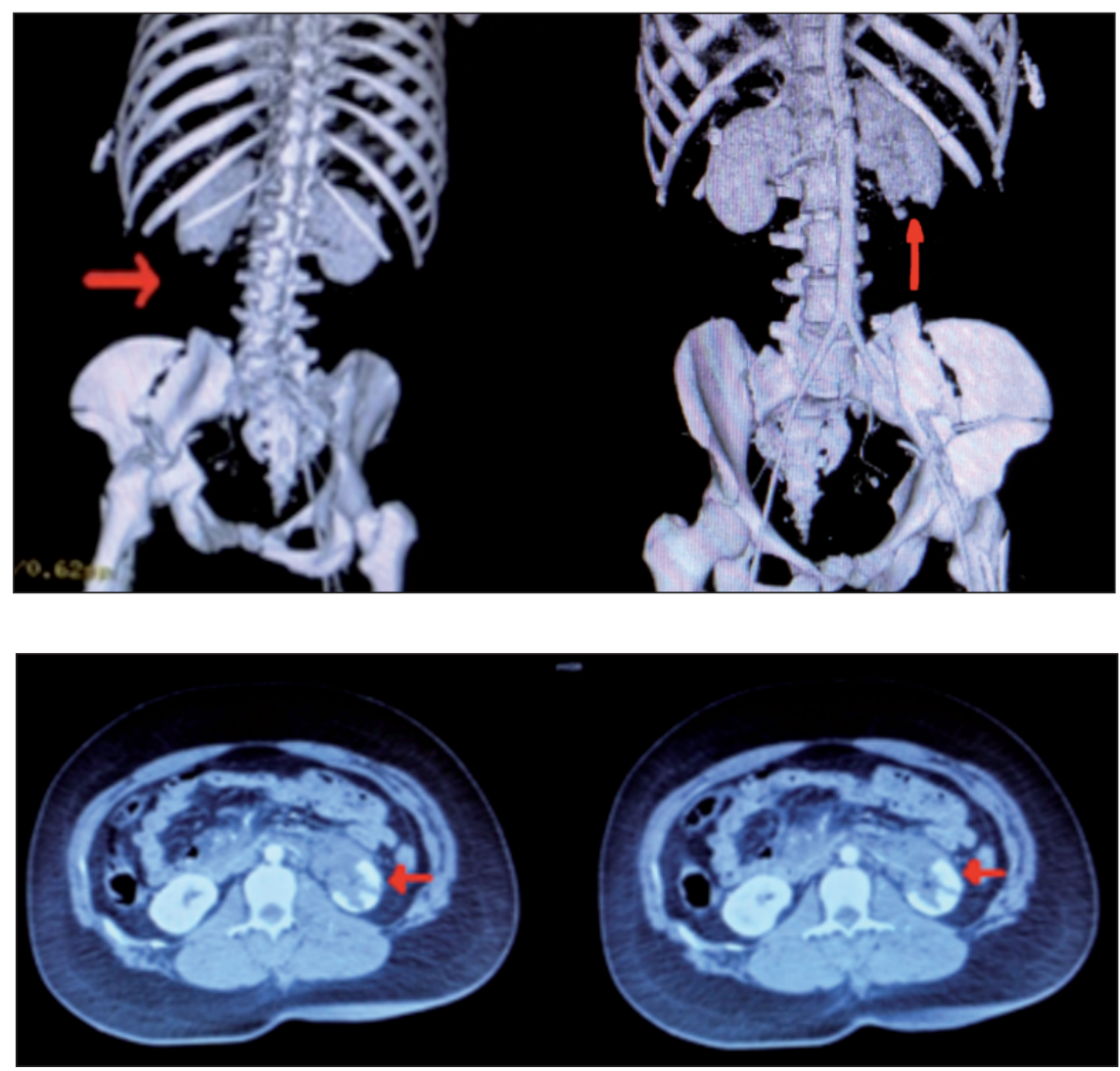

Figure 1.

Multislice 3D CT images

(showed by arrow).
Figure 2.

Multislice CT images transverse axis (showed by arrow).

\section{Conclusion}

Renal traumas are classified under two categories as penetrating and blunt traumas based on their pathogenic mechanisms. Blunt renal traumas can happen as a consequence of primary impact of the trauma and also secondary to concurrent bone fractures (rib fracture and vertebra transverse process fractures etc.). In the literature, a direct relationship between pelvic fracture and renal trauma has not been described so far. We presented a case with grade 3 lower pole injury of the kidney with a fatal course which was presumably correlated with iliac wing fracture.

We think that in cases with pelvic fractures, not only lower urogenital tract, but also upper urinary system should be meticulously examined.
Discussion, and supplementary references are posted on www.aiua.it

\section{REFERENCES}

1. McAninch JW, Carroll PR, Klosterman PW, et al. Renal reconstruction after injury. J Urol. 1991; 145:932-937.

2. Santucci RA, Wessels $H$, Bartsh D, et al. Evaluation and management of renal injuries consensus: Statement of the renal trauma subcommittee. BJU Int. 2004; 93:937.

3. Biurlin MA, Fantus RJ, Mellet MM, Goble SM. Genitourinary injuries in pelvic fracture morbidity and mortality using the National Trauma Data Bank. J Trauma. 2009; 67:1033-1039

4. Abrassart S, Stern R, Peter R. Morbidity associated with isolated iliac wing fractures. J Trauma. 2009; 66:200-203.

\section{Correspondence}

Çaglar Yildirim, MD

Özgür Haki Yüksel, MD (Corresponding Author)

ozgurhaki@gmail.com

Serkan Akan, MD

Ahmet Ürkmez, MD

Ayhan Verit, MD, Prof.

Dept. of Urology, Fatih Sultan Mehmet Research \& Training Hospital Içerenköy/Ataşehir Tr- 34752 Istanbul, Turkey 